\title{
A Reliable \& Novel approach based on Thermodynamic Property estimation of Low to High Salinities Aqueous Sodium Chloride Solutions for Water-Energy Nexus (WEN) Applications
}

Lubna Muzamil Rehman ${ }^{\mathrm{a}}$, Ranjan Dey ${ }^{\mathrm{b}}$, Zhiping Laic, Asim K. Ghosh ${ }^{\mathrm{d}}$, Anirban Roy ${ }^{\mathrm{a}}$

${ }^{a}$ Department of Chemical Engineering, Birla Institute of Technology and Science-Pilani, K.K Birla Goa Campus, Goa, 403726, India

${ }^{b}$ Department of Chemistry, Birla Institute of Technology and Science-Pilani, K.K Goa Birla Campus, Goa, 403726, India

${ }^{c}$ Advanced Membranes and Porous Materials Centre, Division of Physical Science and Engineering, King Abdullah University of Science and Technology (KAUST), Thuwwal, 23955-6900, K.S.A

${ }^{d}$ Membrane Development Section, Chemical Engineering Group, Bhabha Atomic Research Centre, Government of India, Trombay, Mumbai, Maharashtra, 400094, India

*Corresponding Author

Email: anirbanr@goa.bits-pilani.ac.in,

Ph No. 00919830265292 


\section{SUPPLEMENTARY INFORMATION}

Table S1: Tabulation of NaCl-solution properties at different concentrations at $\mathbf{3 0 0 . 1 5 K}$ :

\begin{tabular}{|c|c|c|c|c|c|}
\hline $\begin{array}{c}\text { Concentration } \\
(\mathrm{g} / \mathrm{kg})\end{array}$ & $\begin{array}{c}\text { Viscosity } \\
(\mathrm{kg} / \mathrm{m} . \mathrm{s}) \times 10^{4}\end{array}$ & $\begin{array}{c}\text { Lit. Viscosity* } \\
(\mathrm{kg} / \mathrm{m} . \mathrm{s}) \times 10^{4}\end{array}$ & $\begin{array}{c}\text { Density } \\
\left(\mathrm{kg} / \mathrm{m}^{3}\right)\end{array}$ & $\begin{array}{c}\text { Lit. Density* } \\
\left(\mathrm{kg} / \mathrm{m}^{3}\right)\end{array}$ & $\mathrm{u}(\mathrm{m} / \mathrm{s})$ \\
\hline 0 & 8.53 & 8.53 & 996.50 & 996.42 & 1503.17 \\
\hline 5 & 8.62 & 8.616 & 1000.27 & 1000.16 & 1509.79 \\
\hline 10 & 8.70 & 8.70 & 1003.97 & 1003.94 & 1516.14 \\
\hline 15 & 8.79 & 8.8 & 1007.67 & 1007.77 & 1522.90 \\
\hline 20 & 8.88 & 9.09 & 1011.16 & 1011.60 & 1530.90 \\
\hline 25 & 8.97 & 8.99 & 1014.92 & 1015.38 & 1532.55 \\
\hline 30 & 9.07 & 9.20 & 1018.80 & 1019.16 & 1538.48 \\
\hline 35 & 9.17 & 9.20 & 1022.48 & 1022.96 & 1545.24 \\
\hline 40 & 9.28 & 9.30 & 1026.24 & 1026.76 & 1550.48 \\
\hline 45 & 9.39 & 9.41 & 1030.01 & 1030.56 & 1554.34 \\
\hline 50 & 9.51 & 9.52 & 1033.93 & 1034.36 & 1562.34 \\
\hline 55 & 9.62 & 9.635 & 1037.77 & 1038.14 & 1565.79 \\
\hline 60 & 9.74 & 9.75 & 1041.43 & 1041.92 & 1569.93 \\
\hline 65 & 9.87 & 9.869 & 1045.16 & 1045.75 & 1573.93 \\
\hline 70 & 10.00 & 9.99 & 1049.00 & 1049.58 & 1579.45 \\
\hline 75 & 10.13 & 10.119 & 1053.41 & 1053.38 & 1586.62 \\
\hline 80 & 10.27 & 10.25 & 1056.74 & 1057.18 & 1591.31 \\
\hline 85 & 10.40 & 10.38 & 1060.48 & 1060.96 & 1596.28 \\
\hline 90 & 10.55 & 10.51 & 1064.50 & 1064.74 & 1597.79 \\
\hline 95 & 10.69 & 10.64 & 1068.28 & 1068.54 & 1601.66 \\
\hline 100 & 10.84 & 10.80 & 1072.22 & 1072.34 & 1610.48 \\
\hline
\end{tabular}

*Ref no.1 
Table S2: Tabulation of NaCl-solution properties at different concentrations at $\mathbf{3 0 8 . 1 5 K}$ :

\begin{tabular}{|c|c|c|c|c|c|}
\hline $\begin{array}{c}\text { Concentration } \\
(\mathrm{g} / \mathrm{kg})\end{array}$ & $\begin{array}{c}\text { Viscosity } \\
(\mathrm{kg} / \mathrm{m} . \mathrm{s}) \times 10^{4}\end{array}$ & $\begin{array}{c}\text { Lit. Viscosity* } \\
(\mathrm{kg} / \mathrm{m} . \mathrm{s}) \times 10^{4}\end{array}$ & $\begin{array}{c}\text { Density } \\
\left(\mathrm{kg} / \mathrm{m}^{3}\right)\end{array}$ & $\begin{array}{c}\text { Lit. Density* } \\
\left(\mathrm{kg} / \mathrm{m}^{3}\right)\end{array}$ & $\mathrm{u}(\mathrm{m} / \mathrm{s})$ \\
\hline 0 & 7.24 & 7.25 & 994.03 & 993.94 & 1521.10 \\
\hline 5 & 7.36 & 7.33 & 998.79 & 997.65 & 1525.99 \\
\hline 10 & 7.42 & 7.41 & 1002.44 & 1001.40 & 1530.87 \\
\hline 15 & 7.49 & 7.49 & 1004.09 & 1005.15 & 1535.75 \\
\hline 20 & 7.55 & 7.58 & 1010.73 & 1008.90 & 1540.63 \\
\hline 25 & 7.61 & 7.66 & 1016.77 & 1012.65 & 1545.52 \\
\hline 30 & 7.70 & 7.75 & 1019.03 & 1016.40 & 1551.45 \\
\hline 35 & 7.79 & 7.84 & 1018.68 & 1020.15 & 1557.38 \\
\hline 40 & 7.89 & 7.94 & 1025.33 & 1023.95 & 1563.31 \\
\hline 45 & 7.98 & 8.03 & 1030.98 & 1027.70 & 1569.24 \\
\hline 50 & 8.07 & 8.13 & 1033.15 & 1031.45 & 1575.17 \\
\hline 55 & 8.18 & 8.23 & 1035.28 & 1035.20 & 1578.51 \\
\hline 60 & 8.31 & 8.33 & 1040.92 & 1038.95 & 1581.85 \\
\hline 65 & 8.39 & 8.43 & 1044.57 & 1042.73 & 1585.19 \\
\hline 70 & 8.50 & 8.54 & 1046.22 & 1046.50 & 1588.52 \\
\hline 75 & 8.60 & 8.65 & 1050.08 & 1050.28 & 1591.86 \\
\hline 80 & 8.72 & 8.76 & 1055.52 & 1054.05 & 1597.10 \\
\hline 85 & 8.84 & 8.87 & 1059.17 & 1057.78 & 1602.34 \\
\hline 90 & 8.97 & 8.98 & 1062.82 & 1061.50 & 1607.59 \\
\hline 95 & 9.09 & 9.10 & 1066.47 & 1065.28 & 1612.83 \\
\hline 100 & 9.21 & 9.22 & 1069.10 & 1069.05 & 1618.07 \\
\hline
\end{tabular}

*Ref no.1 
Table S3: Tabulation of NaCl-solution properties at different concentrations at $\mathbf{3 1 5 . 1 5 K}$ :

\begin{tabular}{|c|c|c|c|c|c|}
\hline $\begin{array}{l}\text { Concentration } \\
(\mathrm{g} / \mathrm{kg})\end{array}$ & $\begin{array}{c}\text { Viscosity } \\
(\mathrm{kg} / \mathrm{m} . \mathrm{s}) \\
\mathrm{x} 10^{4} \\
\end{array}$ & $\begin{array}{l}\text { Lit. Viscosity* } \\
(\mathrm{kg} / \mathrm{m} . \mathrm{s}) \times 10^{4}\end{array}$ & $\begin{array}{l}\text { Density } \\
\left(\mathrm{kg} / \mathrm{m}^{3}\right)\end{array}$ & $\begin{array}{c}\text { Lit. Density* } \\
\left(\mathrm{kg} / \mathrm{m}^{3}\right)\end{array}$ & $\mathrm{u}(\mathrm{m} / \mathrm{s})$ \\
\hline 0 & 6.31 & 6.32 & 991.07 & 991.38 & 1533.10 \\
\hline 5 & 6.38 & 6.39 & 994.95 & 995.12 & 1537.93 \\
\hline 10 & 6.46 & 6.46 & 998.83 & 998.86 & 1542.76 \\
\hline 15 & 6.53 & 6.53 & 1002.71 & 1002.56 & 1547.59 \\
\hline 20 & 6.60 & 6.61 & 1006.59 & 1006.26 & 1552.41 \\
\hline 25 & 6.67 & 6.69 & 1010.48 & 1010.00 & 1557.24 \\
\hline 30 & 6.75 & 6.77 & 1013.65 & 1013.74 & 1562.48 \\
\hline 35 & 6.84 & 6.84 & 1017.81 & 1017.44 & 1567.72 \\
\hline 40 & 6.92 & 6.93 & 1021.43 & 1021.22 & 1572.97 \\
\hline 45 & 7.00 & 7.02 & 1025.06 & 1024.92 & 1578.21 \\
\hline 50 & 7.08 & 7.11 & 1026.35 & 1028.62 & 1583.45 \\
\hline 55 & 7.18 & 7.19 & 1032.31 & 1032.36 & 1586.34 \\
\hline 60 & 7.27 & 7.28 & 1035.93 & 1036.10 & 1589.24 \\
\hline 65 & 7.36 & 7.37 & 1039.55 & 1039.84 & 1592.14 \\
\hline 70 & 7.46 & 7.47 & 1043.18 & 1043.58 & 1595.03 \\
\hline 75 & 7.55 & 7.56 & 1043.27 & 1047.32 & 1597.93 \\
\hline 80 & 7.66 & 7.66 & 1050.43 & 1051.06 & 1602.92 \\
\hline 85 & 7.76 & 7.76 & 1054.05 & 1054.76 & 1607.92 \\
\hline 90 & 7.87 & 7.86 & 1057.68 & 1058.46 & 1612.91 \\
\hline 95 & 7.98 & 7.97 & 1061.30 & 1062.20 & 1617.90 \\
\hline 100 & 8.08 & 8.07 & 1063.30 & 1065.94 & 1622.90 \\
\hline
\end{tabular}

*Ref no.1, 2 
Table S4: Comparison of reported and experimentally determined values of osmotic pressure:

\begin{tabular}{|c|c|c|c|c|c|c|}
\hline $\begin{array}{c}\text { Concentration } \\
(\mathrm{g} / \mathrm{kg})\end{array}$ & $\begin{array}{c}\pi_{o} \\
(\mathrm{MPa}) \\
\text { Experimental } \\
300.15 \mathrm{~K}\end{array}$ & $\begin{array}{c}\pi_{o} \\
(\mathrm{MPa}) \\
\text { Lit.* }\end{array}$ & $\begin{array}{c}\pi_{o} \\
(\mathrm{MPa}) \\
\text { Experimental } \\
308.15 \mathrm{~K}\end{array}$ & $\begin{array}{c}\pi_{o} \\
(\mathrm{MPa}) \\
\text { Lit.* }^{*}\end{array}$ & $\begin{array}{c}\pi_{o} \\
(\mathrm{MPa}) \\
\text { Experimental } \\
315.15 \mathrm{~K}\end{array}$ & $\begin{array}{c}\pi_{o} \\
(\mathrm{MPa}) \\
\text { Lit.* }^{*}\end{array}$ \\
\hline 0 & 0 & 0 & 0 & 0 & 0 & 0 \\
\hline 5 & 0.35 & 0.36 & 0.38 & 0.37 & 0.39 & 0.38 \\
\hline 10 & 0.69 & 0.72 & 0.72 & 0.74 & 0.74 & 0.75 \\
\hline 15 & 1.04 & 1.09 & 1.10 & 1.12 & 1.11 & 1.14 \\
\hline 20 & 1.42 & 1.46 & 1.47 & 1.50 & 1.48 & 1.53 \\
\hline 25 & 1.76 & 1.84 & 1.85 & 1.88 & 1.88 & 1.92 \\
\hline 30 & 2.14 & 2.22 & 2.25 & 2.27 & 2.29 & 2.32 \\
\hline 35 & 2.55 & 2.61 & 2.66 & 2.67 & 2.71 & 2.73 \\
\hline 40 & 2.95 & 3.00 & 3.09 & 3.08 & 3.14 & 3.14 \\
\hline 45 & 3.34 & 3.41 & 3.52 & 3.49 & 3.58 & 3.57 \\
\hline 50 & 3.81 & 3.81 & 3.97 & 3.91 & 4.04 & 3.99 \\
\hline 55 & 4.21 & 4.24 & 4.40 & 4.35 & 4.49 & 4.44 \\
\hline 60 & 4.64 & 4.66 & 4.84 & 4.79 & 4.93 & 4.89 \\
\hline 65 & 5.07 & 5.11 & 5.28 & 5.24 & 5.39 & 5.35 \\
\hline 70 & 5.54 & 5.56 & 5.73 & 5.70 & 5.84 & 5.82 \\
\hline 75 & 6.06 & 6.03 & 6.18 & 6.18 & 6.30 & 6.32 \\
\hline 80 & 6.54 & 6.49 & 6.67 & 6.67 & 6.80 & 6.81 \\
\hline 85 & 7.03 & 6.99 & 7.18 & 7.18 & 7.31 & 7.33 \\
\hline 90 & 7.44 & 7.49 & 7.70 & 7.69 & 7.83 & 7.85 \\
\hline 95 & 7.91 & 8.01 & 8.23 & 8.23 & 8.36 & 8.41 \\
\hline 100 & 8.55 & 8.54 & 8.78 & 8.77 & 8.91 & 8.96 \\
\hline
\end{tabular}

*Ref no.1, 2 
Table S5: Comparison of reported and experimentally determined values of vapour pressure:

\begin{tabular}{|c|c|c|c|c|c|c|}
\hline $\begin{array}{l}\text { Concentration } \\
(\mathrm{g} / \mathrm{kg})\end{array}$ & $\begin{array}{c}\pi_{V} \\
(\mathrm{kPa}) \\
\text { Experimental } \\
300.15 \mathrm{~K} \\
\end{array}$ & $\begin{array}{c}\pi_{V} \\
(\mathrm{kPa}) \\
\text { Lit* }\end{array}$ & $\begin{array}{c}\pi_{V} \\
(\mathrm{kPa}) \\
\text { Experimental } \\
308.15 \mathrm{~K} \\
\end{array}$ & $\begin{array}{c}\pi_{V} \\
(\mathrm{kPa}) \\
\mathrm{Lit}^{*}\end{array}$ & $\begin{array}{c}\pi_{V} \\
(\mathrm{kPa}) \\
\text { Experimental } \\
315.15 \mathrm{~K} \\
\end{array}$ & $\begin{array}{c}\pi_{V} \\
(\mathrm{kPa}) \\
\text { Lit* }^{*}\end{array}$ \\
\hline 0 & 3.67 & 3.67 & 5.82 & 5.81 & 8.38 & 8.38 \\
\hline 5 & 3.66 & 3.67 & 5.81 & 5.80 & 8.36 & 8.36 \\
\hline 10 & 3.65 & 3.66 & 5.79 & 5.79 & 8.34 & 8.34 \\
\hline 15 & 3.64 & 3.65 & 5.78 & 5.77 & 8.31 & 8.32 \\
\hline 20 & 3.63 & 3.64 & 5.76 & 5.76 & 8.29 & 8.29 \\
\hline 25 & 3.63 & 3.63 & 5.74 & 5.74 & 8.27 & 8.27 \\
\hline 30 & 3.62 & 3.62 & 5.73 & 5.72 & 8.24 & 8.25 \\
\hline 35 & 3.61 & 3.61 & 5.71 & 5.71 & 8.22 & 8.22 \\
\hline 40 & 3.60 & 3.60 & 5.69 & 5.69 & 8.19 & 8.20 \\
\hline 45 & 3.59 & 3.58 & 5.67 & 5.67 & 8.17 & 8.17 \\
\hline 50 & 3.57 & 3.57 & 5.65 & 5.65 & 8.14 & 8.15 \\
\hline 55 & 3.56 & 3.56 & 5.63 & 5.64 & 8.11 & 8.12 \\
\hline 60 & 3.55 & 3.55 & 5.61 & 5.62 & 8.09 & 8.09 \\
\hline 65 & 3.54 & 3.54 & 5.59 & 5.60 & 8.06 & 8.06 \\
\hline 70 & 3.53 & 3.52 & 5.57 & 5.58 & 8.03 & 8.03 \\
\hline 75 & 3.51 & 3.51 & 5.55 & 5.55 & 8.01 & 8.00 \\
\hline 80 & 3.50 & 3.50 & 5.53 & 5.53 & 7.97 & 7.97 \\
\hline 85 & 3.48 & 3.48 & 5.51 & 5.51 & 7.94 & 7.94 \\
\hline 90 & 3.47 & 3.47 & 5.48 & 5.49 & 7.91 & 7.91 \\
\hline 95 & 3.46 & 3.45 & 5.46 & 5.47 & 7.88 & 7.87 \\
\hline 100 & 3.46 & 3.44 & 5.43 & 5.44 & 7.84 & 7.84 \\
\hline
\end{tabular}

*Ref no.1 
Table S6: Tabulation of the experimental results obtained of different Thermo-acoustical properties at different temperatures.

\begin{tabular}{|c|c|c|c|c|c|c|}
\hline $\begin{array}{c}\text { Temperature } \\
\text { (K) }\end{array}$ & $\begin{array}{c}\text { Concentration } \\
(\mathrm{g} / \mathrm{kg})\end{array}$ & $\begin{array}{c}\mathbf{L}_{\mathbf{f}} \\
\mathrm{x} 10^{11}(\mathrm{~m})\end{array}$ & $\begin{array}{c}\mathbf{Z} \\
\left(\mathrm{kg} / \mathrm{m}^{2} \mathrm{~s}\right)\end{array}$ & $\begin{array}{c}\mathbf{V}_{\mathbf{f}} \\
\times 10^{8} \\
\left(\mathrm{~m}^{3} / \mathrm{mol}\right)\end{array}$ & $\begin{array}{c}\boldsymbol{\tau} \\
\mathrm{x} 10^{13}(\mathrm{~s})\end{array}$ & $\mathbf{B} / \mathbf{A}$ \\
\hline \multirow{5}{*}{300.15} & 0 & 4.35 & 1497911.31 & 2.018 & 5.0512 & 7.483 \\
\hline & 25 & 4.228 & 1555413.105 & 1.976 & 5.019 & 7.33 \\
\hline & 50 & 4.109 & 1615347.376 & 1.913 & 5.021 & 7.181 \\
\hline & 75 & 4.01 & 1671363.687 & 1.824 & 5.093 & 7.063 \\
\hline & 100 & 3.915 & 1726793.434 & 1.726 & 5.199 & 6.951 \\
\hline \multirow{5}{*}{308.15} & 0 & 4.367 & 1512022.461 & 2.632 & 4.191 & 7.389 \\
\hline & 25 & 4.246 & 1574526.6 & 2.561 & 4.17 & 7.264 \\
\hline & 50 & 4.133 & 1630539.724 & 2.475 & 4.189 & 7.118 \\
\hline & 75 & 4.058 & 1673174.383 & 2.343 & 4.305 & 7.038 \\
\hline & 100 & 3.96 & 1729869.441 & 2.2204 & 4.387 & 6.916 \\
\hline \multirow{5}{*}{315.15} & 0 & 4.394 & 1519417.434 & 3.266 & 3.612 & 7.327 \\
\hline & 25 & 4.284 & 1573553.483 & 3.157 & 3.629 & 7.206 \\
\hline & 50 & 4.18 & 1625172.138 & 3.036 & 3.668 & 7.078 \\
\hline & 75 & 4.109 & 1667071.912 & 2.865 & 3.779 & 7.01 \\
\hline & 100 & 4.01 & 1725621.035 & 2.714 & 3.847 & 6.894 \\
\hline
\end{tabular}




\section{Additional plots and relations:}
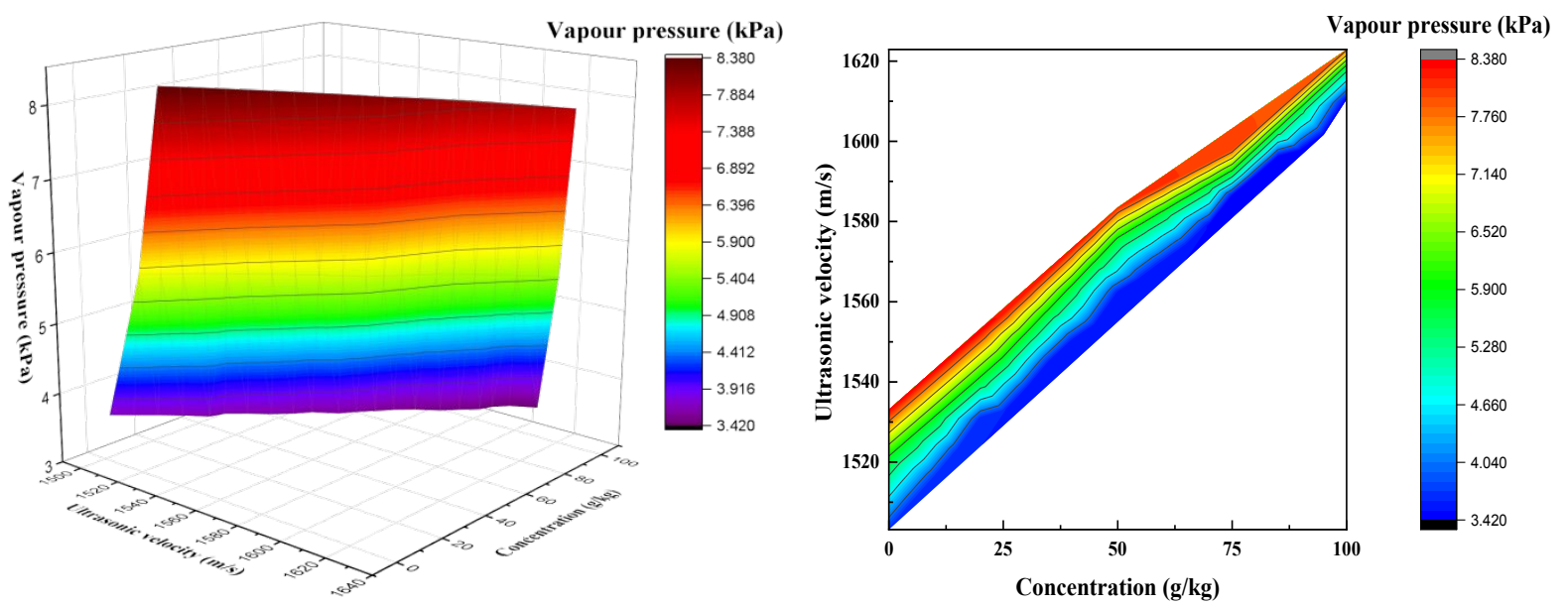

Figure S1: Surface fit of vapour pressure as a function of ultrasonic velocity and concentration
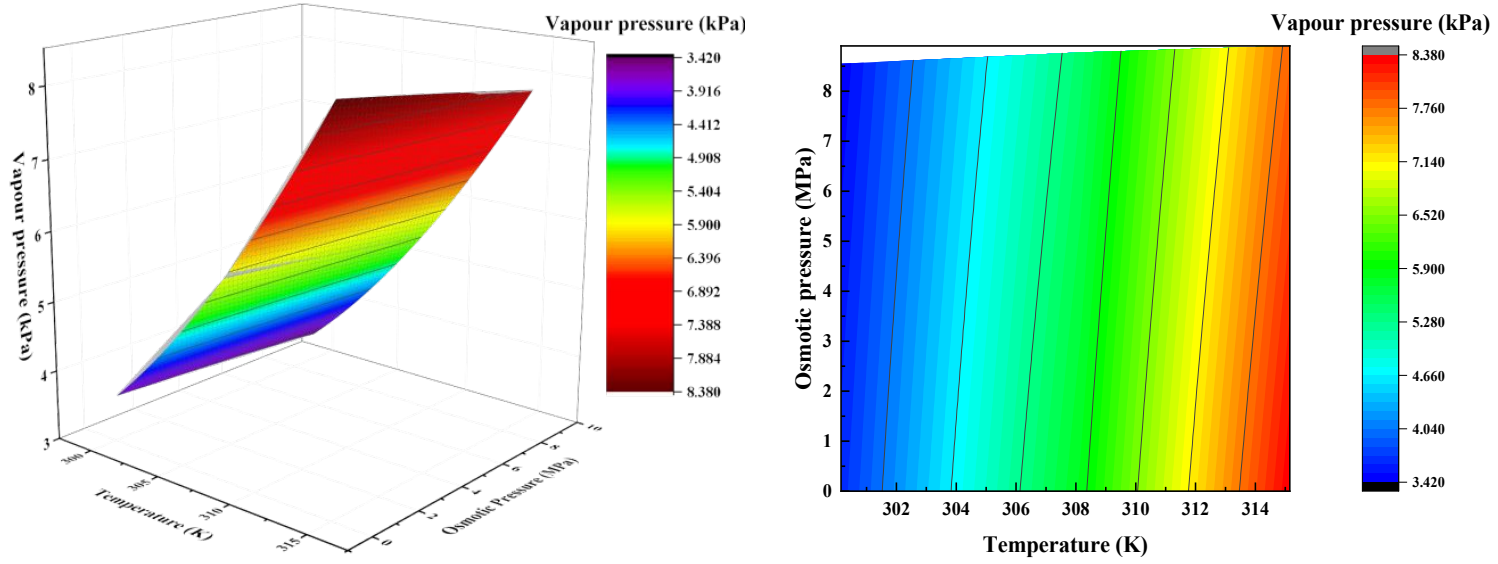

Figure S2: Surface fit of vapour pressure as a function of osmotic pressure and temperature.

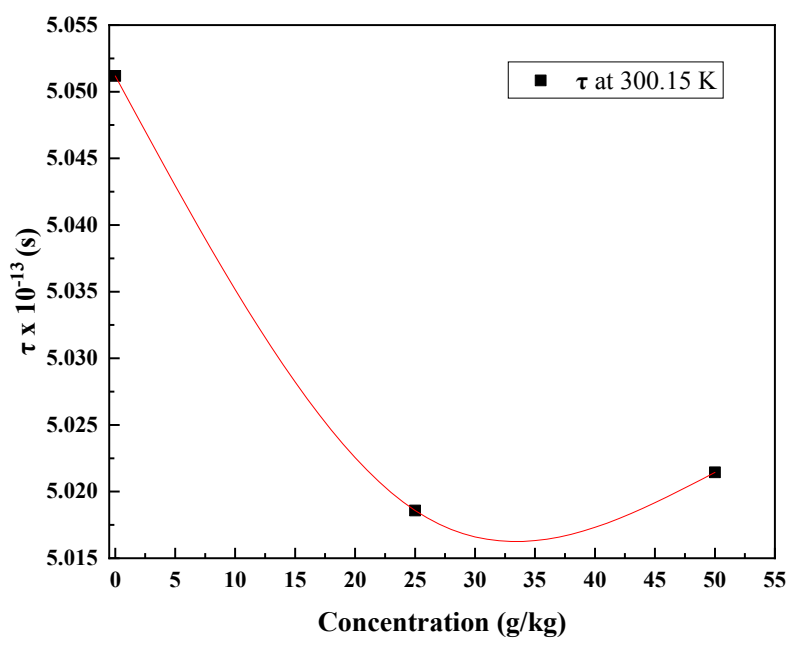

Figure S3: Expanded view of relaxation time plotted at $300.15 \mathrm{~K}$ : shows a clear minima between 25$40 \mathrm{~g} / \mathrm{kg}$. 


\section{References:}

1. Nayar, K. G.; Sharqawy, M. H.; Banchik, L. D.; Lienhard, J. H. Thermophysical Properties of Seawater: A Review and New Correlations That Include Pressure Dependence. Desalination 2016, 390, 1-24

2. Mistry, K. H.; Lienhard, J. H. Generalized Least Energy of Separation for Desalination and Other Chemical Separation Processes. Entropy 2013, 15 (6), 2046-2080 